\title{
Using the cervical range of motion (CROM) device to assess head repositioning accuracy in individuals with cervical radiculopathy in comparison to neck- healthy individuals
}

Johanna Wibault, Jacques Vaillant, Nicolas Vuillerme, Åsa Dedering and Anneli Peolsson

\author{
Linköping University Post Print
}

\section{Tweet}

N.B.: When citing this work, cite the original article.

Original Publication:

Johanna Wibault, Jacques Vaillant, Nicolas Vuillerme, Åsa Dedering and Anneli Peolsson, Using the cervical range of motion (CROM) device to assess head repositioning accuracy in individuals with cervical radiculopathy in comparison to neck- healthy individuals, 2013, Manual Therapy, (18), 5, 403-409.

http://dx.doi.org/10.1016/j.math.2013.02.004

Copyright: Elsevier http://www.elsevier.com/

Postprint available at: Linköping University Electronic Press http://urn.kb.se/resolve?urn=urn:nbn:se:liu:diva-98660 
Using the cervical range of motion (CROM) device to assess head repositioning accuracy in individuals with cervical radiculopathy in comparison to neck- healthy individuals

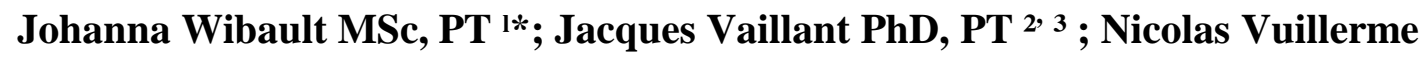
$\mathrm{PhD}^{3}$; Åsa Dedering PhD, PT ${ }^{4}$; Anneli Peolsson PhD, PT ${ }^{1}$

${ }^{1}$ Department of Medical and Health Sciences, Division of Physiotherapy, Faculty of Health Sciences, Linköping University, Linköping, Sweden

${ }^{2}$ Ecole de kinésithérapie du centre Hospitalier Universitaire de Grenoble, France

${ }^{3}$ AGIM (AGeing Imaging Modeling) Laboratory, FRE 3405, CNRS-UJF-UPMFEPHE, Faculty of Medicine, La Tronche, France

${ }^{4}$ Division of Physiotherapy, Department of Neurobiology, Care sciences and Society, Department of physical therapy, Karolinska University Hospital, Stockholm, Sweden

Corresponding author:

Johanna Wibault* MSc, PT, PhD stud

Department of Medical and Health Sciences

Division of Physiotherapy

Faculty of Health Sciences

Linköping University

SE-58183 Linköping

Sweden

Tel: +46101031765

Fax: +46-10 1031706

E-mail: Johanna.Wibault@liu.se 
Key words: Neck pain, Radiculopathy, Head repositioning accuracy, Measurement

\begin{abstract}
$\underline{\text { ABSTRACT }}$
This study had two purposes: to compare head repositioning accuracy (HRA) using the cervical range of motion (CROM) device between individuals with cervical radiculopathy caused by disc disease (CDD; $\mathrm{n}=71)$ and neck- healthy individuals ( $\mathrm{n}=$ 173); and to evaluate the test- retest reliability of the CROM device in individuals with CDD, and criterion validity between the CROM device and a laser in neck-healthy individuals, with quantification of measurement errors. Parameters of reliability and validity were expressed with intra- class- correlation coefficients (ICCs), and measurement errors with standard error of measurement (SEM) and Bland Altman limits of agreement. HRA (Mdn, IQR) differed significantly between individuals with CDD and neck- healthy individuals after rotation right $2.7^{\circ}(6.0), 1.7^{\circ}(2.7)$; and rotation left $2.7^{\circ}(3.3), 1.3^{\circ}(2.7)(p<0.021)$; 31\% of individuals with CDD were classified as having impairment in HRA. The test- retest reliability of the CROM device in individuals with CDD showed ICCs of $0.79-0.85$, and SEMs of $1.4^{\circ}-2^{\circ}$. The criterion validity between the CROM device and the laser in neck-healthy individuals showed ICCs of $0.43-0.91$ and SEMs of $0.8^{\circ}-1.3^{\circ}$. The results support the use of the CROM device for quantifying HRA impairment in individuals with CDD in clinical practice; however, criterion validity between the CROM device and a laser in neckhealthy individuals was questionable. HRA impairment in individuals with CDD may be important to consider during rehabilitation and evaluated with the criterion established with the CROM device in neck-healthy individuals.
\end{abstract}




\section{INTRODUCTION}

Sensorimotor function relates to the control of posture and movements (Treleaven, 2008). The contribution of cervical muscles to sensorimotor function has been emphasized with regards to the density of muscle spindles that reflect a well-developed proprioceptive system (Dutia, 1991; Boyd-Clark et al., 2002), and cervical muscles play a major role in motor control of the head and neck (Dutia, 1991; Peterson, 2004; Armstrong et al., 2008), eye movements (Karlberg et al., 1991), and bipedal posture during quiet standing (Vuillerme et al., 2005).

The ability to reposition the head in a neutral position after active head movements has been used to indirectly assess impairment in sensorimotor function originating from the neck (Revel et al., 1991; Loudon et al., 1997; Heikkila and Wenngren, 1998; Kristjansson et al., 2003; Treleaven et al., 2003). Larger than typical errors in head repositioning accuracy (HRA) have been reported in individuals with neck disorders (Revel et al., 1991; Loudon et al., 1997; Kristjansson et al., 2003; Treleaven et al., 2003); although the results are controversial (Rix and Bagust, 2001; Hill et al., 2009); without a consensus on the best method (Strimpakos, 2011). The original test used a laser to assess HRA (Revel et al., 1991), a method that has since been widely used (Heikkila and Wenngren, 1998; Vuillerme et al., 2008; Pinsault and Vuillerme, 2010) and that exhibits good reliability (Pinsault et al., 2008a) and validity (Pinsault et al., 2008b; Roren et al., 2009). As an alternative method for assessment of HRA, the Cervical Range of Motion (CROM) device has been used in several studies with reported good reliability (Loudon et al., 1997; Dumas et al., 2001; Uremovic et al., 2007). 
Individuals with cervical radiculopathy caused by disc disease (CDD) display reduced physical functioning and overall health (Peolsson et al., 2002; Daffner et al., 2003; Ylinen et al., 2003; Peolsson and Kjellman, 2007). Assessment of HRA is recommended in individuals with neck pain (Humphreys, 2008; Treleaven, 2008; Kristjansson and Treleaven, 2009), but to our knowledge, studies reporting this assessment have not previously been carried out in individuals with CDD.

The CROM device possesses several advantages for clinical practice because it can be managed by one rater and requires no advanced time-consuming calculations, but the test- retest reliability and measurement error of the CROM device for assessment of HRA in individuals with CDD are unknown (Mokkink et al., 2010). Knowledge about assessment of HRA with the CROM device in neck- healthy individuals is also limited (Loudon et al., 1997), and the device has not been compared to a laser which might be considered the gold standard for clinical practice (Roren et al., 2009).

The present study had two specific purposes. The first was to compare assessment of HRA with the CROM device between individuals with CDD and neck-healthy individuals. The second was to evaluate the test- retest reliability of the CROM device in individuals with CDD, and criterion validity between the CROM device and a laser in neck- healthy individuals, with quantification of measurement errors. 


\section{$\underline{\text { METHODS }}$}

\section{Participants}

The present experimental study included one sample of individuals with CDD, and two samples of neck- healthy individuals. Participation was voluntary, and participants provided written informed consent. The regional ethical review board approved the study.

Individuals with cervical radiculopathy

Individuals with CDD were referred to neck surgery and consecutively recruited from a neurosurgery department at a University Hospital in Sweden. Inclusion criteria were age 18- 65 years and an association between clinical findings and verified CDD on MRI. Individuals with previous surgery, earlier fracture or luxation of the cervical spine, malignity or spinal tumor, myelopathy, systematic disease, diagnosis of fibromyalgia or generalized myofascial pain, persistent or recurring severe back pain, diagnosed psychiatric disorders, alcohol or drug addiction, or lack of familiarity with the Swedish language were excluded. Seventy-one individuals with CDD participated in the study (38 men; 33 women; mean age 50 years, standard deviation (SD) 10.0 years) (Table 1). Twenty-four individuals (14 men; 10 women; mean age 51 years, (SD) 8.4 years) (Table 1) also contributed to the evaluation of the test- retest reliability and measurement error of the CROM device in individuals with CDD.

\section{Neck- healthy individuals}

Individuals permanently employed at a hospital were stratified according to sex and age and randomly selected (computerized random list developed by a statistician) to be 
asked to volunteer in the comparative study of HRA assessment using the CROM device between individuals with CDD and neck-healthy individuals (640 individuals; 340 men; 300 women). A total of 149 individuals (75 men; 74 women) met the inclusion criteria of no self-reported current neck disorders (score on the Neck Disability Index (NDI) < 20\% (Fairbank et al., 1980), pain on the visual analogue scale (VAS) $\leq 10 \mathrm{~mm}$ (Croft et al., 1998), and no recurrent neck or low back pain, inflammatory joint disease, or other systemic disease during the last three years; 10 of the recruited men were unable to attend the testing. The sample was filled with 34 individuals (employees and students from a university) to include at least 80 men and 80 women (20 individuals in each of the following age intervals: 25- 34, 35- 44, 45- 54 and 55- 64 years) (Peolsson et al., 2007). The final sample included 173 individuals (86 women; 87 men; mean age 44 years, SD 12.0) (Table 1). A convenient sample of 12 neck-healthy individuals (10 women; 2 men; mean age 42 years, (SD) 8.5 years) (Table 1) was recruited from employees at a university for participation in the criterion validity study between the CROM device and laser. The neck- healthy individuals differed significantly from individuals with CDD in age, body mass index (BMI) and level of physical activity ( $\mathrm{p}<0.001)$.

\section{Measurements}

\section{Background data}

Individuals completed the NDI (Vernon and Mior, 1991), reported pain intensity on the VAS (Harms-Ringdahl et al., 1986), and estimated their daily physical activity and their weekly practice of exercise, sport, and open-air activities during the preceding12 
months. Answers to the two questions were interpreted on the basis of a four- point scale (1= inactivity, $2=$ low activity, $3=$ moderate activity, 4= high activity) in accordance with a previous study (Peolsson et al., 2007). A self-reporting measure for assessment of physical activity has been evaluated accurate and reliable when compared to objective quantification (Babor et al., 2004).

\section{Assessment of head repositioning accuracy with the CROM device}

Assessment of HRA with the CROM device was performed according to a previous protocol (Loudon et al., 1997). Individuals were seated in an upright position on a stool with no backrest with the CROM device on their head, and both feet on the floor. A self-chosen neutral head position (NHP) was established as the starting and reference positions, and the CROM device was adjusted to zero in the primary plane of movement. Individuals were instructed to close their eyes, memorize the starting position, actively rotate their head $30^{\circ}$, and reposition their head to the starting position with a maximum of precision but no requirements for speed. HRA was measured as the difference in degrees in the primary plane of movement between the starting and the return positions because this protocol previously proved valid for detecting differences in HRA between neck-healthy individuals and individuals with neck disorders (Revel et al., 1991; Treleaven et al., 2003). Individuals performed three repetitions within $60 \mathrm{~s}$ in each rotation direction, providing a total of six trials. The assessments were performed by two physiotherapists and three students from the Department of Physiotherapy, all of whom were previously trained in the testing procedure. 
Test- retest reliability and measurement error of the CROM device in individuals with cervical radiculopathy

Test- retest reliability and measurement error for the CROM device in individuals with CDD $(n=24)$ were determined by two repeated measurements. One physiotherapist performed the measurements within a 1-hour interval.

Criterion validity and measurement error between the CROM device and laser in neckhealthy individuals

Criterion validity and measurement error between the CROM device and laser in neckhealthy- individuals $(\mathrm{n}=12)$ were evaluated with the CROM device and laser simultaneously assembled on each individual's head according to previously described protocols (Revel et al., 1991; Loudon et al., 1997) (Figure 1). The starting and reference NHPs for the laser were marked on plotting paper mounted on a wall $1 \mathrm{~m}$ away, and the CROM device was concurrently adjusted to zero in the primary plane of movement. Individuals were instructed to switch on the laser with a handheld button when they returned to the starting position. Two raters participated in the study to allow simultaneous assessment of HRA with the CROM device and laser. One rater marked the location of the return positions on the plotting paper and the second rater registered the degrees of error in HRA on the CROM device. Individuals performed a total of eight consecutive repetitions in each rotation direction, providing a total of 16 trials. NHP was adjusted before changing rotation direction (Pinsault et al., 2008a). The instruments were randomly allocated between the two raters; however, the raters assessed both instruments after switching places. The repositioning marks on the plotting paper represented the global error for each trial and were described with (x;y) 
coordinates with the coordinates on the $\mathrm{x}$-axis representing the repositioning error in the primary plane of movement. The distance in centimeters between NHP and the coordinate on the $\mathrm{x}$-axis for each return position was transformed to a measure in degrees with the use of the trigonometric formula $\tan ^{-1}(\mathrm{OP} / \mathrm{OH})$ where $\mathrm{OP}$ was the distance of $1 \mathrm{~m}$ between the laser and the wall with the plotting paper.

\section{$\underline{\text { Statistical analysis }}$}

HRA was calculated in individuals as the mean of the absolute errors of repeated repositioning trials in both rotation directions, and expressed for groups with median (Mdn), interquartile range (IQR), mean value and standard deviation (SD). Since the results of the Kolomogorov- Smirnov test showed the data not to meet the assumptions of normality, non- parametric statistics were used. For difference between age groups, the Kruskal- Wallis test was used. For differences between sex and between individuals with CDD and neck-healthy individuals the Mann- Whitney U was used, and the Wilcoxon signed rank test for differences between rotation directions. A criterion for impairment in HRA is necessary both for group comparison in research and individual assessment in clinical practice, and a cut-off value was determined using the upper $90^{\text {th }}$ percentile of the mean of the maximal error in HRA in neck-healthy individuals (Peolsson and Kjellman, 2007). Maximal error in HRA was the larger error measured for every individual after rotation to either the right or the left. Values above the cut-off were classified as impairment in HRA. The correlations between HRA and age, sex, body height, body weight, BMI, and physical activity in neck-healthy individuals, as well as the correlations between maximal error in HRA, pain, and NDI in individuals 
with CDD were estimated with Spearman's correlation coefficients. Statistical significance was set at $p<0.05$. To allow comparison with previous studies where a parametrical approach was used, intra- class- correlation coefficients (ICCs absolute agreement and consistency agreement, two-way random effects model, average measures) and 95\% CI for ICC were used to evaluate the test- retest reliability of the CROM device in individuals with CDD and criterion validity between the CROM device and laser in neck-healthy individuals. Measurement errors were expressed with standard error of measurement (SEM) (de Vet et al., 2006); and Bland Altman 95\% limits of agreement (LOAs) (Figures 2 and 3) (Bland and Altman, 1986). 


\section{$\underline{\text { RESULTS }}$}

Head repositioning accuracy in neck-healthy individuals

HRA (Mdn, IQR) in neck-healthy individuals ( $\mathrm{n}=173$ ) was after rotation to the right $1.7^{\circ}$ (2.7), and after rotation to the left $1.3^{\circ}$ (2.7) (Table 2). Maximal error in HRA was $2.7^{\circ}(2.8)$. No significant difference was found between age groups ( $\left.p=0.28-0.42\right)$, between men and women $(p=0.15-0.26)$, or between rotation directions ( $p=0.59)$ (Table 2). The criterion for impairment in HRA was $6.7^{\circ}$. No significant correlation of HRA with age, sex, body height, body weight, BMI, or physical activity was found for any rotation direction $(\mathrm{r}=-0.11-0.12 ; p=0.12-0.99)$.

Head repositioning accuracy in individuals with cervical radiculopathy HRA differed significantly between individuals with CDD $(\mathrm{n}=71)$ and neck-healthy individuals $(\mathrm{n}=173)$ for rotation to the right $(p=0.012)$, rotation to the left $(p=0.021)$, and maximal error in HRA $(p<0.001)$. HRA (Mdn, IQR) in individuals with CDD was after rotation to the right $2.7^{\circ}(6.0)$, and after rotation to the left $2.7^{\circ}$ (3.3) (Table 2). Maximal error in HRA was $4.0^{\circ}$ (5.3). There was no significant difference between rotation direction ( $p=0.62)$ (Table 2$)$, or between men and women ( $p=0.17-0.31$ ). Twenty-two individuals with CDD (31\%) were classified as having impairment in HRA. No significant correlation between maximal error in HRA and pain $(\mathrm{r}=0.07, p=$ $0.55)$, or disability $(\mathrm{r}=0.12, p=0.34)$ was found. 
Test- retest reliability and measurement error of the CROM in individuals with cervical radiculopathy

The test- retest reliability of the CROM device for assessment of HRA in individuals with CDD $(n=24)$ showed for rotation to the right ICC of 0.79 (95\% CI: 0.50; 0.91), SEM $2^{\circ}$ with $95 \%$ LOA $(-5.57$; 5.68); and for rotation to the left ICC of 0.85 (95\% CI: 0.64; 0.93), SEM $1.4^{\circ}$ with $95 \%$ LOA (-3.89; 4.03).

Criterion validity and measurement error between the CROM device and laser in neckhealthy individuals

The criterion validity between the CROM device and laser in neck-healthy individuals $(\mathrm{n}=12)$ showed ICCs of $0.43-0.91$, and SEM values were $0.8^{\circ}-1.3^{\circ}$ (Table 3). 


\section{DISCUSSION}

The main purpose of the present study was to compare assessment of HRA using the CROM device between individuals with CDD and neck-healthy individuals. The observed statistically significant difference in HRA is consistent with findings from previous studies that included individuals with other types of neck disorders (Revel et al., 1991; Loudon et al., 1997; Heikkila and Wenngren, 1998; Kristjansson et al., 2003; Treleaven et al., 2003; Roren et al., 2009). The current values for HRA also are similar to those reported in a study comparing individuals with whiplash- associated disorders to neck-healthy individuals in which mean HRA was respectively $3.6^{\circ}$ and $2^{\circ}$ after rotation to the right and $4.1^{\circ}$ and $2.5^{\circ}$ after rotation to the left (Treleaven et al., 2003). Comparison of our results for HRA with those of other studies should nevertheless be done with caution considering the use of different assessment methods and statistical analysis (Loudon et al., 1997; Roren et al., 2009), and that no other study was identified that included individuals with CDD.

Our results for HRA with the CROM device in neck- healthy individuals are consistent with those of other studies reporting no effect of age and sex on HRA (DemailleWlodyka et al., 2007). However, the influence of age on HRA is controversial (Teng et al., 2007; Vuillerme et al., 2008) and thus could still represent a source of bias in the present study considering that individuals with CDD were significantly older. Individuals with CDD had higher BMIs, were less physically active, and there was a small imbalance in sex compared with the neck-healthy individuals, but these differences are not expected to have influenced the results since HRA has not been associated with demographic variables. 
The frequency of HRA impairment reported in individuals with CDD in the present study was lower than that reported in a study that included individuals with whiplashassociated disorders and that classified 59\% with HRA impairment (Treleaven et al., 2006). Results should nevertheless be compared with caution considering the use of different assessment methods and criterion for HRA impairment. HRA impairment in individuals with neck pain has commonly been associated with dysfunction in sensory input from cervical muscle spindles (Revel et al., 1991; Treleaven et al., 2003; Treleaven et al., 2006), although the underlying mechanisms remains unknown (Peterson, 2004; Humphreys, 2008). Impairment in HRA in the present study may reflect compromised sensorimotor function in individuals with CDD. HRA impairment has been associated with dizziness (Treleaven et al., 2003), problems in oculomotor and balance function (Heikkila and Wenngren, 1998; Treleaven et al., 2006), and pain in the upper cervical region (Treleaven et al., 2011). No association between background variables and HRA was found in the present study, and the interpretation of our findings is limited.

To minimize the effect of fatigue and pain on HRA in individuals with CDD (Pinsault and Vuillerme, 2010; Strimpakos, 2011), and because many patients with CDD have limited cervical range of motion (Peolsson et al., 2002), the range of motion during active cervical rotation was standardized to $30^{\circ}$ (Uremovic et al., 2007) in this study. In addition, the number of trials was limited to three in both rotation directions (Loudon et al., 1997; Treleaven et al., 2003; Treleaven et al., 2006). More repetitions have been recommended to improve the reliability of HRA measurement (Swait et al., 2007; Pinsault et al., 2008a), but more repetitions could possibly have increased pain in 
individuals with CDD and negatively influenced the measurements. If a maximal cervical range of motion had been used, the results for HRA as well as the frequency of individuals classified with impairment in HRA might have been different. A difference in the number of participants in the two samples is a limitation and was directly related to the number of individuals with CDD recruited in the study.

ICC values, SEM, and 95\% LOA should be interpreted as characteristics of an instrument used in a population (Weir, 2005). Our results suggest that the CROM device can be used to quantify impairment in HRA with some acceptable level of reliability in individuals with CDD (Weir, 2005), and in a manner comparable to a laser in individuals with neck pain (ICC = 0.68) (Roren et al., 2009). However, the SEM data indicate a level of variability with the CROM device that may overlook small impairments in HRA and question the use of the CROM device to evaluate true changes in HRA over time (Weir, 2005). The criterion validity between the CROM device and laser in neck-healthy individuals was questionable. Given the small samples, parameters of reliability, validity, and measurement error reported in the present study should be interpreted with caution. More extensive methodological studies considering measurement properties of the CROM device for the assessment of HRA may be necessary. 


\section{$\underline{\text { CONCLUSION }}$}

Significantly larger errors in HRA were measured with the CROM device in individuals with CDD compared to neck-healthy individuals; 31\% of individuals with CDD were classified as having impairment in HRA. The test- retest reliability and measurement error reported for the CROM device supports its use for quantifying HRA impairments with some acceptable level of reliability in individuals with CDD; however, the criterion validity between the CROM device and laser in neck-healthy individuals was questionable. One clinical implication of the present study is that HRA impairment in individuals with CDD might be important to consider during rehabilitation, and may be evaluated with the criterion established with the CROM device in neck-healthy individuals.

\section{ACKNOWLEDGEMENT}

The authors acknowledge financial support from The Swedish Research Council, The Swedish Society of Medicine, and the Medical Research Council of Southeast Sweden. The authors thank all individuals who contributed to the present study, as well as the students Nadja Börjesson, Rasmus Elb and Sofia Finnkvist from the Department of Physiotherapy at the University of Linköping for their help in collecting data from neckhealthy individuals. 


\section{$\underline{\text { REFERENCES }}$}

Armstrong B, McNair P, Taylor D. Head and neck position sense. Sports Medicine 2008; 38: 101-17.

Babor T F, Sciamanna C N, Pronk N P. Assessing multiple risk behaviors in primary care. Screening issues and related concepts. American Journal of Preventive Medicine 2004; 27: 42-53.

Bland J M, Altman D G. Statistical methods for assessing agreement between two methods of clinical measurement. Lancet 1986; 1: 307-10.

Boyd-Clark L C, Briggs C A, Galea M P. Muscle spindle distribution, morphology, and density in longus colli and multifidus muscles of the cervical spine. Spine 2002; 27: 694-701.

Croft P R, Macfarlane G J, Papageorgiou A C, Thomas E, Silman A J. Outcome of low back pain in general practice: a prospective study. British Medical Journal 1998; 316: 1356-9.

Daffner S D, Hilibrand A S, Hanscom B S, Brislin B T, Vaccaro A R, Albert T J. Impact of neck and arm pain on overall health status. Spine 2003; 28: 2030-5.

de Vet H C, Terwee C B, Knol D L, Bouter L M. When to use agreement versus reliability measures. Journal of Clinical Epidemiology 2006; 59: 1033-9.

Demaille-Wlodyka S, Chiquet C, Lavaste J F, Skalli W, Revel M, Poiraudeau S. Cervical range of motion and cephalic kinesthesis: ultrasonographic analysis by age and sex. Spine 2007; 32: E254-61.

Dumas J P, Arsenault A B, Boudreau G, Magnoux E, Lepage Y, Bellavance A, Loisel P. Physical impairments in cervicogenic headache: traumatic vs. nontraumatic onset. Cephalalgia 2001; 21: 884-93.

Dutia M B. The muscles and joints of the neck: their specialisation and role in head movement. Progress in Neurobiology 1991; 37: 165-78.

Fairbank J C, Couper J, Davies J B, O'Brien J P. The Oswestry low back pain disability questionnaire. Physiotherapy 1980; 66: 271-3.

Harms-Ringdahl K, Carlsson A M, Ekholm J, Raustorp A, Svensson T, Toresson H G. Pain assessment with different intensity scales in response to loading of joint structures. Pain 1986; 27: 401-11.

Heikkila H V, Wenngren B I. Cervicocephalic kinesthetic sensibility, active range of cervical motion, and oculomotor function in patients with whiplash injury. Archives of Physical Medicine and Rehabilitation 1998; 79: 1089-94.

Hill R, Jensen P, Baardsen T, Kulvik K, Jull G, Treleaven J. Head repositioning accuracy to neutral: a comparative study of error calculation. Manual Therapy 2009; 14: 110-4.

Humphreys B K. Cervical outcome measures: testing for postural stability and balance. Journal of Manipulative and Physiological Therapeutics 2008;31:540-6.

Karlberg M, Magnusson M, Johansson R. Effects of restrained cervical mobility on voluntary eye movements and postural control. Acta Oto-laryngologica 1991; 111: 664-70.

Kristjansson E, Dall'Alba P, Jull G. A study of five cervicocephalic relocation tests in three different subject groups. Clinical Rehabilitation 2003; 17: 768-74. 
Kristjansson E, Treleaven J. Sensorimotor function and dizziness in neck pain: implications for assessment and management. The Journal of Orthopaedic and Sports Physical Therapy 2009; 39: 364-77.

Loudon J K, Ruhl M, Field E. Ability to reproduce head position after whiplash injury. Spine 1997; 22: 865-8.

Mokkink L B, Terwee C B, Patrick D L, Alonso J, Stratford P W, Knol D L, Bouter L $\mathrm{M}$, de Vet H C. The COSMIN study reached international consensus on taxonomy, terminology, and definitions of measurement properties for healthrelated patient-reported outcomes. Journal of Clinical Epidemiology 2010; 63: 737-45.

Peolsson A, Almkvist C, Dahlberg C, Lindqvist S, Pettersson S. Age- and sex-specific reference values of a test of neck muscle endurance. Journal of Manipulative and Physiological Therapeutics 2007; 30: 171-7.

Peolsson A, Kjellman G. Neck muscle endurance in nonspecific patients with neck pain and in patients after anterior cervical decompression and fusion. Journal of Manipulative and Physiological Therapeutics 2007; 30: 343-50.

Peolsson A, Vavruch L, Oberg B. Disability after anterior decompression and fusion for cervical disc disease. Advances in Physiotherapy 2002; 4: 111-24.

Peterson B W. Current approaches and future directions to understanding control of head movement. Progress in Brain Research 2004; 143: 369-81.

Pinsault N, Fleury A, Virone G, Bouvier B, Vaillant J, Vuillerme N. Test-retest reliability of cervicocephalic relocation test to neutral head position. Physiotherapy Theory Practice 2008a; 24: 380-91.

Pinsault N, Vuillerme N. Degradation of cervical joint position sense following muscular fatigue in humans. Spine 2010; 35: 294-7.

Pinsault N, Vuillerme N, Pavan P. Cervicocephalic relocation test to the neutral head position: assessment in bilateral labyrinthine-defective and chronic, nontraumatic neck pain patients. Archives of Physical Medicine and Rehabilitation 2008b; 89: 2375-8.

Revel M, Andre-Deshays C, Minguet M. Cervicocephalic kinesthetic sensibility in patients with cervical pain. Archives of Physical Medicine and Rehabilitation 1991; 72: 288-91.

Rix G D, Bagust J. Cervicocephalic kinesthetic sensibility in patients with chronic, nontraumatic cervical spine pain. Archives of Physical Medicine and Rehabilitation 2001; 82: 911-9.

Roren A, Mayoux-Benhamou M A, Fayad F, Poiraudeau S, Lantz D, Revel M. Comparison of visual and ultrasound based techniques to measure head repositioning in healthy and neck-pain subjects. Manual Therapy 2009; 14: 2707.

Strimpakos N. The assessment of the cervical spine. Part 1: Range of motion and proprioception. Journal of Bodywork and Movement Therapies 2011;15:114-24.

Swait G, Rushton A B, Miall R C, Newell D. Evaluation of cervical proprioceptive function: optimizing protocols and comparison between tests in normal subjects. Spine 2007; 32: E692-701.

Teng C C, Chai H, Lai D M, Wang S F. Cervicocephalic kinesthetic sensibility in young and middle-aged adults with or without a history of mild neck pain. Manual Therapy 2007; 12: 22-8. 
Treleaven J. Sensorimotor disturbances in neck disorders affecting postural stability, head and eye movement control. Manual Therapy 2008;13:2-11.

Treleaven J, Clamaron-Cheers C, Jull G. Does the region of pain influence the presence of sensorimotor disturbances in neck pain disorders? Manual Therapy 2011; 16 : 636-40.

Treleaven J, Jull G, LowChoy N. The relationship of cervical joint position error to balance and eye movement disturbances in persistent whiplash. Manual Therapy 2006; 11: 99-106.

Treleaven J, Jull G, Sterling M. Dizziness and unsteadiness following whiplash injury: characteristic features and relationship with cervical joint position error. Journal of Rehabilitation Medicine 2003; 35: 36-43.

Uremovic M, Cvijetic S, Pasic M B, Seric V, Vidrih B, Demarin V. Impairment of proprioception after whiplash injury. Collegium Antropologicum 2007; 31: 823-7.

Weir J P. Quantifying test-retest reliability using the intraclass correlation coefficient and the SEM. Journal of Strength and Conditioning Research 2005; 19: 231-40.

Vernon H, Mior S. The Neck Disability Index: a study of reliability and validity. Journal of Manipulative and Physiological Therapeutics 1991; 14: 409-15.

Vuillerme N, Pinsault N, Bouvier B. Cervical joint position sense is impaired in older adults. Aging Clinical and Experimental Research 2008; 20: 355-8.

Vuillerme N, Pinsault N, Vaillant J. Postural control during quiet standing following cervical muscular fatigue: effects of changes in sensory inputs. Neuroscience Letters 2005; 378: 135-9.

Ylinen J J, Savolainen S, Airaksinen O, Kautiainen H, Salo P, Hakkinen A. Decreased strength and mobility in patients after anterior cervical diskectomy compared with healthy subjects. Archives of Physical Medicine and Rehabilitation 2003; 84: 1043-7. 
Table 1: Background information for individuals with cervical radiculopathy caused by disc disease (CDD) and neck-healthy individuals

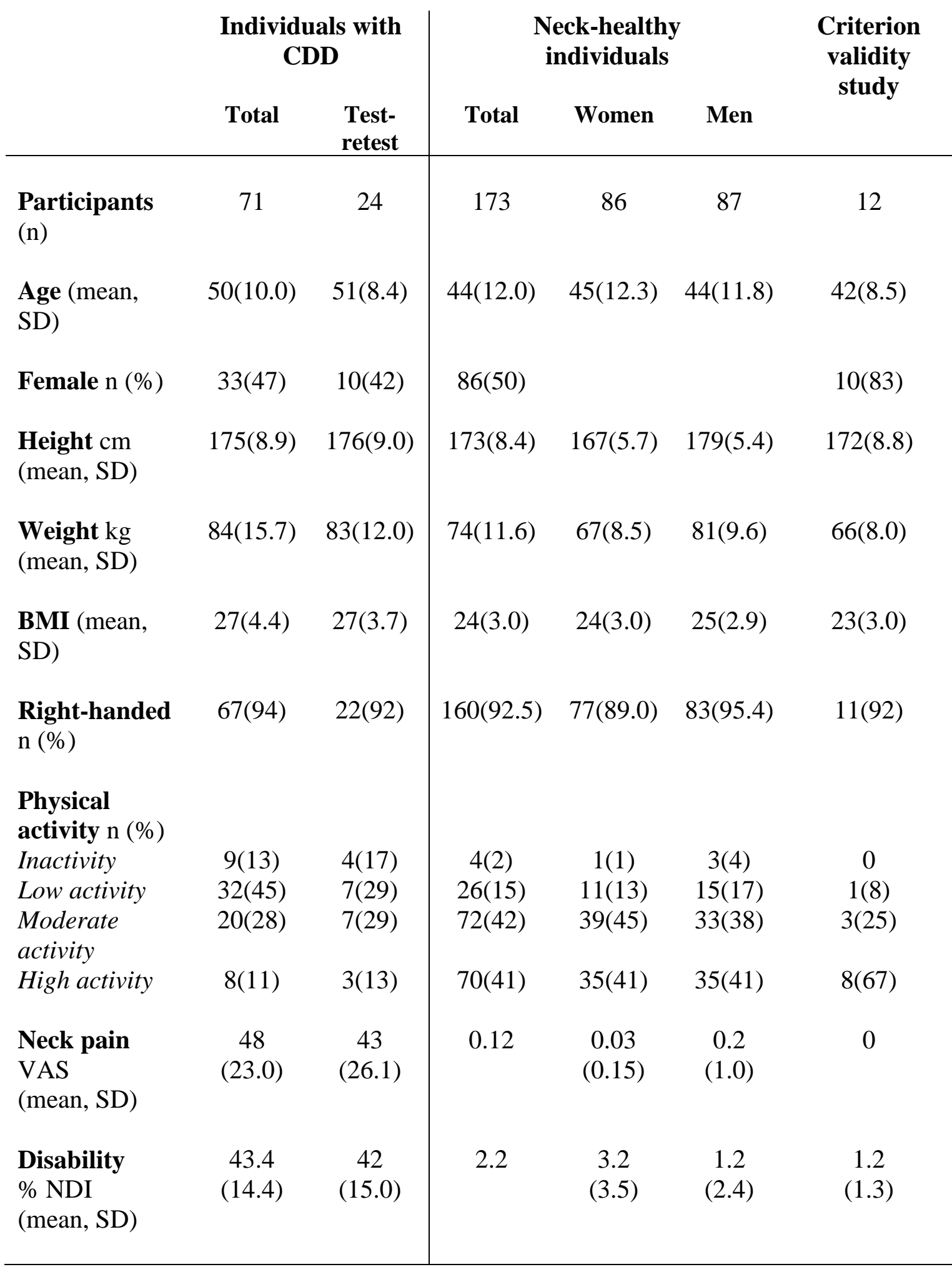

${ }^{\mathrm{a}}$ BMI: Body Mass Index 
${ }^{\mathrm{b}}$ VAS: Visual Analogue Scale for rating pain intensity

${ }^{\mathrm{c}}$ NDI: Neck Disability Index 
Table 2: Head repositioning accuracy (HRA) after rotation to the right and the left expressed with median and interquartile range (Mdn, IQR), mean value and standard deviation (Mean, SD) in neck-healthy individuals and in individuals with cervical radiculopathy caused by disc disease (CDD)

\begin{tabular}{|c|c|c|c|c|c|c|}
\hline \multirow[b]{2}{*}{ Participants } & \multirow[b]{2}{*}{$\mathbf{n}$} & \multicolumn{2}{|c|}{ HRA rotation right } & \multicolumn{2}{|c|}{ HRA rotation left } & \multirow[t]{2}{*}{$\mathrm{p}$} \\
\hline & & $\begin{array}{l}\text { Mdn } \\
\text { (IQR) }\end{array}$ & $\begin{array}{l}\text { Mean } \\
\text { (SD) }\end{array}$ & $\begin{array}{l}\text { Mdn } \\
\text { (IQR) }\end{array}$ & $\begin{array}{l}\text { Mean } \\
\text { (SD) }\end{array}$ & \\
\hline \multicolumn{7}{|l|}{$\begin{array}{l}\text { Neck-healthy } \\
\text { individuals }\end{array}$} \\
\hline Total sample & 173 & $1.7(2.7)$ & $2.2(2.1)$ & $1.3(2.7)$ & $2.4(2.4)$ & 0.59 \\
\hline Women & 86 & $1.7(2.7)$ & $2.5(2.5)$ & $1.7(3.0)$ & $2.7(2.7)$ & \multirow[t]{5}{*}{0.64} \\
\hline 20-34 years & 21 & $1.3(2.7)$ & $2.7(3.2)$ & $1.7(3.0)$ & $3.1(2.9)$ & \\
\hline 35-44 years & 20 & $1.7(2.0)$ & $2.1(2.0)$ & $1.3(3.0)$ & $2.3(2.4)$ & \\
\hline $45-54$ years & 24 & $2.3(3.8)$ & $2.5(2.0)$ & $1.7(3.2)$ & $2.9(2.9)$ & \\
\hline 55-65 years & 21 & $1.7(3.2)$ & $2.7(2.6)$ & $1.7(2.2)$ & $2.4(2.5)$ & \\
\hline Men & 87 & $1.7(2.0)$ & $1.9(1.7)$ & $1.3(2.7)$ & $2.0(2.0)$ & 0.69 \\
\hline 20-34 years & 23 & $1.3(2.7)$ & $1.9(1.3)$ & $1.3(2.7)$ & $2.0(1.8)$ & \\
\hline 35-44 years & 24 & $1.0(2.7)$ & $1.6(1.7)$ & $0.7(2.7)$ & $1.8(2.3)$ & \\
\hline 45-54 years & 20 & $1.3(1.7)$ & $1.8(1.5)$ & $1.3(2.7)$ & $1.8(1.7)$ & \\
\hline 55-65 years & 20 & $1.7(2.5)$ & $2.4(2.3)$ & $2.3(2.5)$ & $2.8(2.3)$ & \\
\hline \multicolumn{7}{|c|}{ Individuals with CDD } \\
\hline Total sample & 71 & $2.7(6.0)$ & $3.8(3.6)$ & $2.7(3.3)$ & $3.5(3.5)$ & 0.62 \\
\hline Women & 33 & $3.7(5.3)$ & $4.3(3.4)$ & $2.7(2.0)$ & $3.4(2.8)$ & 0.18 \\
\hline Men & 38 & $2.3(5.7)$ & $3.4(3.7)$ & $2.0(6.2)$ & $3.6(4.0)$ & 0.79 \\
\hline
\end{tabular}


Table 3: Criterion validity between the CROM device and laser in neck-healthy individuals $(\mathrm{n}=12)$ for three and eight repetitions expressed with intra- classcorrelation coefficients (ICC), 95\% confidence interval (CI), standard error of measurement (SEM), and Bland Altman 95\% limits of agreements (LOA).

\begin{tabular}{|c|c|c|c|c|}
\hline \multirow[t]{2}{*}{$\begin{array}{l}\text { Criterion validity } \\
\text { CROM- laser }\end{array}$} & \multicolumn{2}{|c|}{$\begin{array}{c}\text { Rater a (CROM), } \\
\text { rater b (laser) }\end{array}$} & \multicolumn{2}{|c|}{$\begin{array}{c}\text { Rater a (laser), } \\
\text { rater b (CROM) }\end{array}$} \\
\hline & $\begin{array}{c}\text { HRA rr } \\
3 \text { rep } \\
\text { (8 rep) }\end{array}$ & $\begin{array}{c}\text { HRA rl } \\
3 \text { rep } \\
\text { (8rep) }\end{array}$ & $\begin{array}{c}\text { HRA rr } \\
3 \text { rep } \\
\text { (8rep) }\end{array}$ & $\begin{array}{c}\text { HRA rl } \\
3 \text { rep } \\
\text { (8rep) }\end{array}$ \\
\hline ICC & $0.87(0.81)$ & $0.83(0.91)$ & $0.63(0.43)$ & $0.84(0.76)$ \\
\hline $95 \%$ CI & $\begin{array}{c}0.53,0.96 \\
(0.35,0.95)\end{array}$ & $\begin{array}{c}0.40,0.95 \\
(0.70,0.98)\end{array}$ & $\begin{array}{c}-0.30,0.89 \\
(-0.99,0.84)\end{array}$ & $\begin{array}{c}0.45,0.95 \\
(0.18,0.93)\end{array}$ \\
\hline SEM ( $\left.{ }^{\circ}\right)$ & $0.9(1)$ & $1(0.8)$ & $1.3(1.3)$ & $1(1.2)$ \\
\hline $95 \%$ LOA & $\begin{array}{c}-1.74,3.31 \\
(-2.2,3.5)\end{array}$ & $\begin{array}{c}-1.60,4.08 \\
(-1.5,3.1)\end{array}$ & $\begin{array}{c}-2.98,4.28 \\
(-3.1,4.0)\end{array}$ & $\begin{array}{c}-0.39,3.85 \\
(-1.5,4.9)\end{array}$ \\
\hline
\end{tabular}




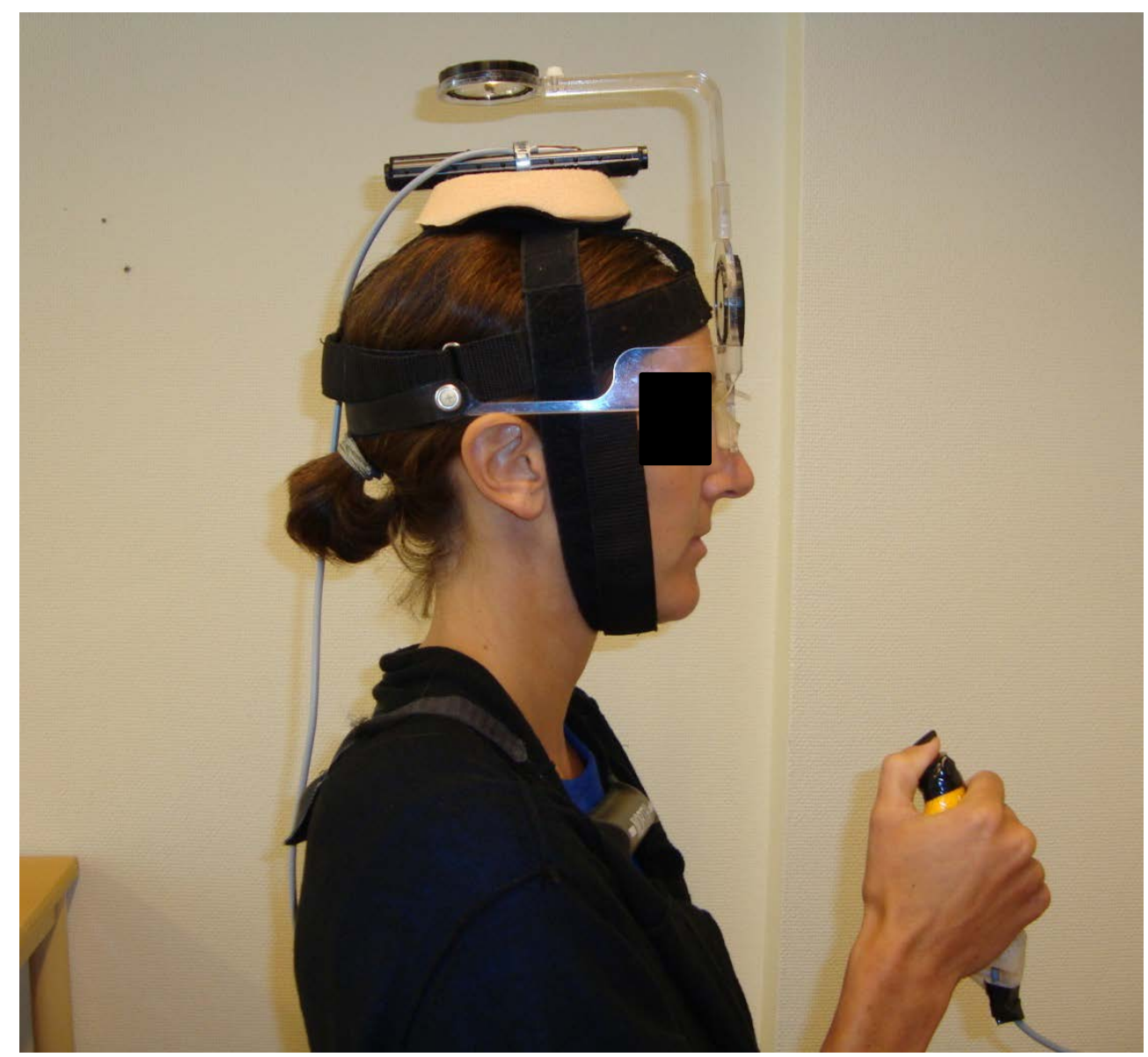

Figure 1: Evaluation of criterion validity between the CROM device and a laser for head repositioning accuracy (HRA) assessment in neck- healthy individuals by simultaneous montage of the CROM device and a laser on the individual's head. 


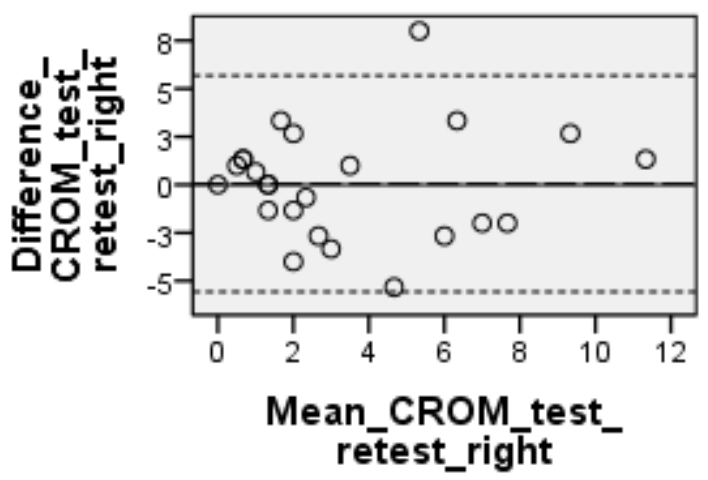

a) Test- retest reliability of the CROM after rotation to the right

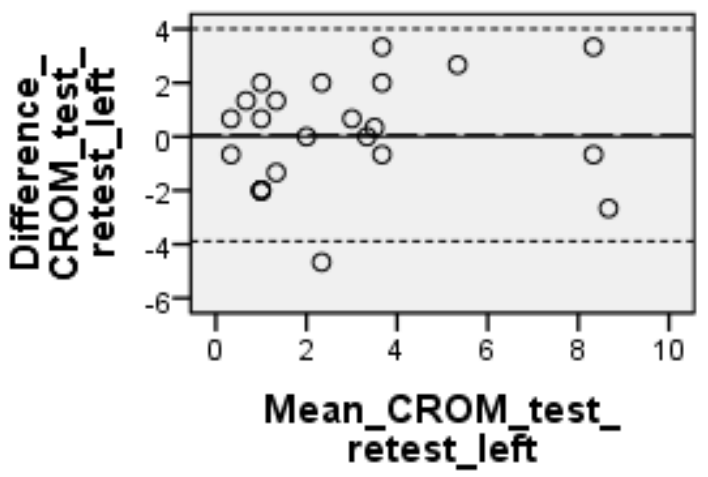

b) Test- retest reliability of the CROM after rotation to the left

Figure 2: Bland Altman plots with 95\% limits of agreement (LOA) for the test- retest reliability of head repositioning accuracy (HRA) assessment with the CROM device after rotation to the right (a) and to the left (b) in individuals with cervical radiculopathy $(\mathrm{CDD}, \mathrm{n}=24)$. The difference between the two measurements is presented on the $\mathrm{y}$ axis, and the mean of the two measurements on the x-axis, with the lines showing the observed average difference, 95\% LOA, and $(y=0)$ the perfect average difference. 


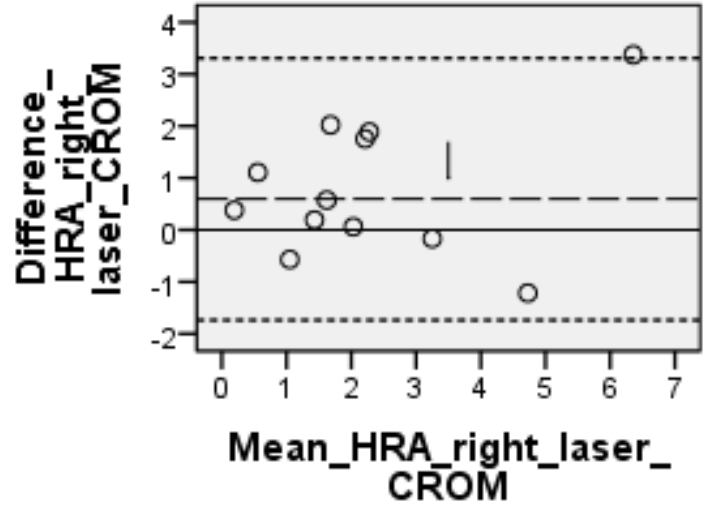

a) HRA after rotation to the right: CROM (rater a), laser (rater b)

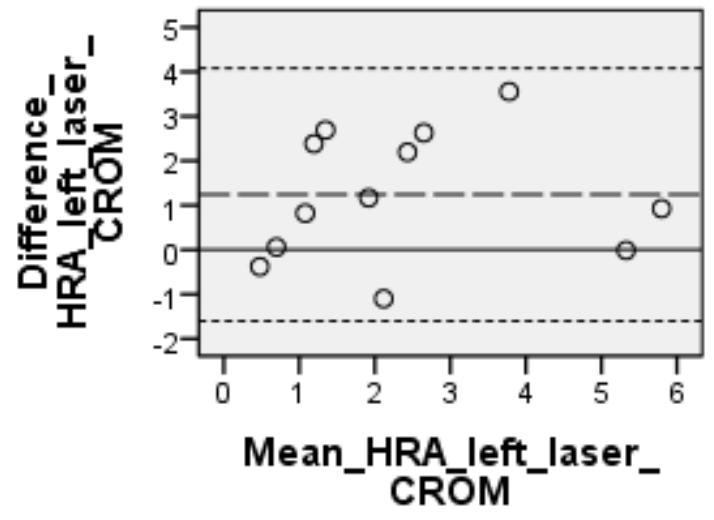

c) HRA after rotation to the left: CROM (rater a), laser (rater b)

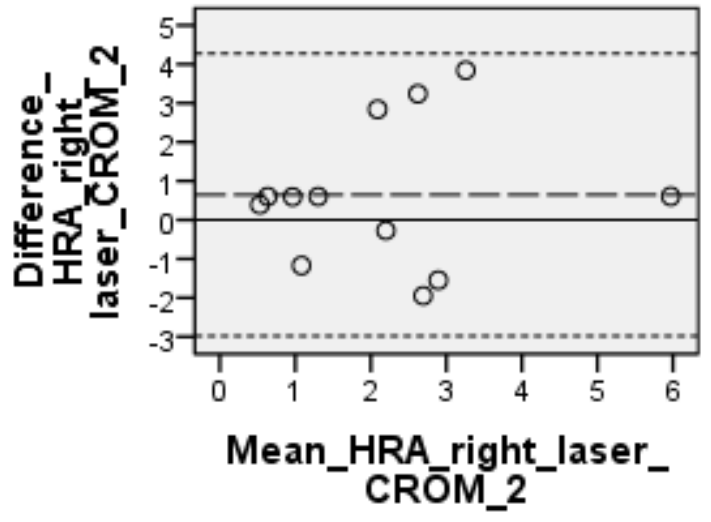

b) HRA after rotation to the right: CROM (rater b), laser (rater a)

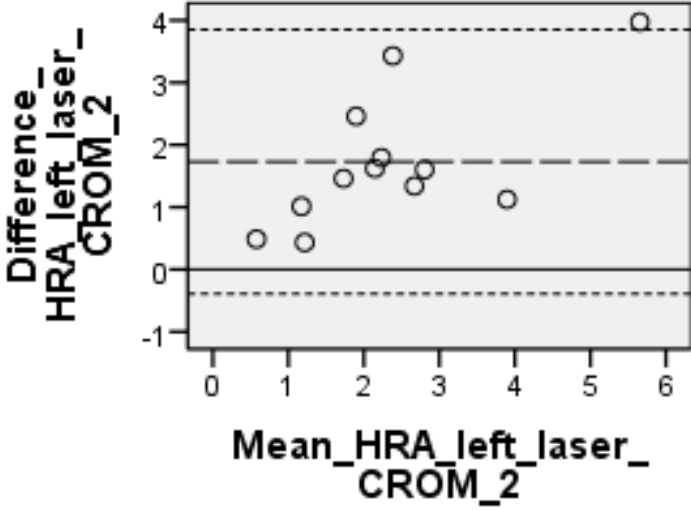

d) HRA after rotation to the left: CROM (rater b), laser (rater a)

Figure 3: Bland Altman plots with 95\% limits of agreement (LOA) for criterion validity between the CROM device and a laser for head repositioning accuracy (HRA) assessment after rotation to the right (a, b) and to the left (c, d) in neck-healthy individuals $(\mathrm{n}=12)$. Difference between the two measurements is presented on the $\mathrm{y}$ axis, the mean of the two measurements on the $\mathrm{x}$-axis, and the lines representing the observed average difference, 95\% LOA and $(y=0)$ the perfect average difference. Results are presented for the two raters assessing each instrument. 